\title{
ESTADOS POTENCIALES EN MÉXICO PARA LA PRODUCCIÓN Y CONSUMO DE FRIJOL BIOFORTIFICADO CON HIERRO Y ZINC
}

\section{POTENTIAL STATES IN MEXICO FOR PRODUCTION AND CONSUMPTION OF BEANS BIOFORTIFIED WITH IRON AND ZINC}

\author{
Rocío Ramírez-Jaspeado ${ }^{1 *}$, Natalia Palacios-Rojas ${ }^{1}$, \\ Marilia Nutti ${ }^{2}$ y Salomón Pérez ${ }^{2}$
}

'Centro International de Mejoramiento de Maíz y Trigo, Texcoco, Edo. de México, México. ${ }^{2}$ Centro Internacional de Agricultura Tropical, HarvestPlus, Cali, Colombia

*Autor de correspondencia (ramirezrocio67@hotmail.com)

\section{RESUMEN}

A nivel mundial una de cada tres personas padece al menos una forma de malnutrición como desnutrición, sobrepeso u obesidad. En México, a pesar de las intervenciones de salud pública para incrementar la ingesta de micronutrientes como el zinc, la desnutrición crónica aún representa 13.6 $\%$, mientras $23.3 \%$ sufre anemia, causada principalmente por deficiencia de hierro. El frijol (Phaseolus vulgaris) es el segundo cultivo que más se produce y consume, después del maíz (Zea mays $L$.), con una ingesta promedio anual per capita de $10 \mathrm{~kg}$. La biofortificación podría contribuir a reducir la desnutrición en zonas rurales y marginales al incrementar la ingesta de micronutrientes sin modificar los patrones de consumo. El objetivo de este estudio fue priorizar los estados del país para la intervención con frijol biofortifcado con hierro y zinc, según su mayor impacto potencial, mediante el Índice de Priorización para Biofortificación y las condiciones de biofortificación, basados en producción, consumo y deficiencia de micronutrientes. Chiapas, Oaxaca, Guerrero, Veracruz, Campeche y Tabasco deberían ser priorizados para la intervención con frijol negro biofortificado, mientras que Baja California Sur y Sonora para frijol pinto bioforificado; Guanajuato y Tlaxcala son prioritarios para frijol claro biofortificado.

Palabras clave: Biofortificación, desnutrición, hierro, leguminosas, micronutrientes, zinc.

\section{SUMMARY}

Worldwide, one in three people suffers from at least one form of malnutrition such as under-nutrition, overweight or obesity. In Mexico, despite public health interventions to increase the intake of micronutrients such as zinc, chronic malnutrition still represents $13.6 \%$, while $23.3 \%$ suffer from anemia, caused mainly by iron deficiency. Common beans (Phaseolus vulgaris) is the second most produced and consumed crop after maize (Zea mays L.), with an average annual per capita intake of $10 \mathrm{~kg}$. Biofortification could contribute to reduce malnutrition in rural and marginal areas by increasing the intake of micronutrients without modifying consumption patterns. The objective of this study was to prioritize the states of the country for intervention with beans biofortified with iron and zinc, according to their greatest potential impact, through the Prioritization Index for Biofortification and biofortification conditions, based on production, consumption and micronutrient deficiency. Chiapas, Oaxaca, Guerrero, Veracruz, Campeche and Tabasco should be prioritized for intervention with biofortified black beans, while Baja California Sur and Sonora for biofortified pinto beans; Guanajuato and Tlaxcala are priorities for biofortified light beans.
Index words: Biofortification, iron, malnutrition, micronutrients, vegetables, zinc.

\section{INTRODUCCIÓN}

Según la Organización Mundial de la Salud (OMS, 2018), una de cada tres personas en el mundo padece al menos una forma de malnutrición, como desnutrición, sobrepeso u obesidad, lo que afecta a 155 millones de niños menores de cinco años, provocando efectos adversos en el desarrollo físico y cognitivo (FAO et al. 2017), en el mediano y largo plazo en el rendimiento escolar e intelectual, el desarrollo de sus capacidades, el rendimiento en el trabajo y su ingreso laboral cuando son adultos (Gutiérrez et al., 2012).

En México, la incidencia del retraso en el crecimiento ha disminuido en los últimos 25 años, gracias a programas de suplementación con micronutrientes como ácido fólico, vitamina A y hierro, así como los programas sociales que otorgan productos alimenticios fortificados con micronutrientes (Gutiérrez et al., 2012), aunque los indicadores son desiguales entre regiones. De acuerdo con la clasificación de INEGI, las regiones del país se integran de la siguiente forma: 1) Centro: Ciudad de México, Hidalgo, Estado de México, Morelos, Puebla y Tlaxcala; 2) Centro Occidente: Aguascalientes, Colima, Guanajuato, Jalisco, Michoacán, Nayarit, Querétaro, San Luis Potosí y Zacatecas; 3) Noreste: Chihuahua, Coahuila, Durango, Nuevo León y Tamaulipas; 4) Noroeste: Baja California, Baja California Sur, Sonora y Sinaloa; y 5) Sureste: Campeche Chipas, Guerrero, Oaxaca, Quintana Roo, Tabasco, Veracruz y Yucatán Actualmente 14 de cada 100 preescolares (1.5 millones) presentan indicadores de retraso en el crecimiento en estados como Chiapas, Guerrero y Oaxaca, de la región Sureste y Puebla de la región centro del país, con una prevalencia de desnutrición promedio de entre 20 y $32 \%$ 
(WHO, 2013; Gutiérrez et al., 2012).

La dieta deficiente en hierro es una de las causas más frecuentes de anemia en el país, con $23.3 \%$ de la población afectada (Gutiérrez et al., 2012). En estados como Campeche, Guerrero, Guanajuato y el Estado de México se concentran índices de 35, 30, 28 y $27 \%$, respectivamente (INSP, 2012) (Figura 1). Aproximadamente $18.5 \%$ de las mujeres embarazadas de entre 12 y 49 años padecen de anemia derivado de las necesidades de hierro del feto (OMS, 2011), mientras que esta tasa es de $16.2 \%$ en mujeres no embarazas de 40 a 49 años, resultado de cambios metabólicos de la edad, la baja ingesta de hierro biodisponible o una dieta inadecuada (Olivares y Walter, 2003).

A pesar de los logros alcanzados, $45.2 \%$ de los hogares rurales y $40.6 \%$ de los hogares urbanos experimentan preocupación por el acceso a los alimentos y 35.4 y 26.2 $\%$, respectivamente, consumen una dieta insuficiente en cantidad, calidad y, en casos extremos, de hambre debido al escaso acceso a los alimentos, disponibilidad de dinero u otros recursos (Gutiérrez et al., 2012). La incorporación de zinc y hierro depende en buena parte de consumir alimentos de origen animal como pescado, pollo, res, cerdo y vegetales como legumbres y cereales de grano entero (Latham, 2002).

El cultivo de frijol (Phaseolus vulgaris L.) ha formado parte de la dieta desde tiempos prehispánicos (Leyva et al., 2016), es uno de los cultivos más comúnmente encontrados en los sistemas productivos de la milpa, conceptualizada ésta como un sistema agrícola con maíz y con otras especies que son cosechadas o toleradas en temporal. Es el segundo cultivo más importante después del maíz (Zea mays L.) en el sector agrícola mexicano (De los Santos-Ramos et al., 2017; Prieto-Cornejo et al., 2019), y representa no sólo una tradición de producción, sino de consumo, especialmente en las áreas rurales (MárquezQuiroz et al., 2015). Según Espinoza-García et al. (2016), se han encontrado variedades nativas de frijol en Oaxaca con contenidos de zinc entre 3.3 y $6.1 \mathrm{mg} 100 \mathrm{~g}^{-1}$, y de hierro entre 3.9 y $7.4 \mathrm{mg} 100 \mathrm{~g}^{-1}$; datos similares a los reportados por Moraghan y Grafton (2001), Prolla et al. (2010) y Martínez et al. (2013) para frijol en Centro y Sudmérica, aunque los cultivares actuales tienen en promedio $2.79 \mathrm{mg}$ de zinc y $5.5 \mathrm{mg}$ de hierro por cada $100 \mathrm{~g}$ de grano crudo (Leyva et al., 2016).

Actualmente, el frijol se cultiva en los 32 estados del país; en 2017 se destinó 7.8 \% de la superficie agrícola total cosechada para la producción de este grano, lo cual corresponde a 1.6 millones de hectáreas (88\% de temporal y $12 \%$ riego) (SIAP, 2018), representado por más de 70 variedades (CONABIO, 2012). El volumen de producción alcanzado para el mismo año ascendió a 1.18 millones de toneladas, agrupados en tres grupos, 36 \% frijoles claros, $31 \%$ negros y $29 \%$ pintos (SIAP, 2018). En 2017, el $74 \%$ de la producción se concentró en seis estados: Zacatecas, Sinaloa, Durango, Chihuahua, Nayarit

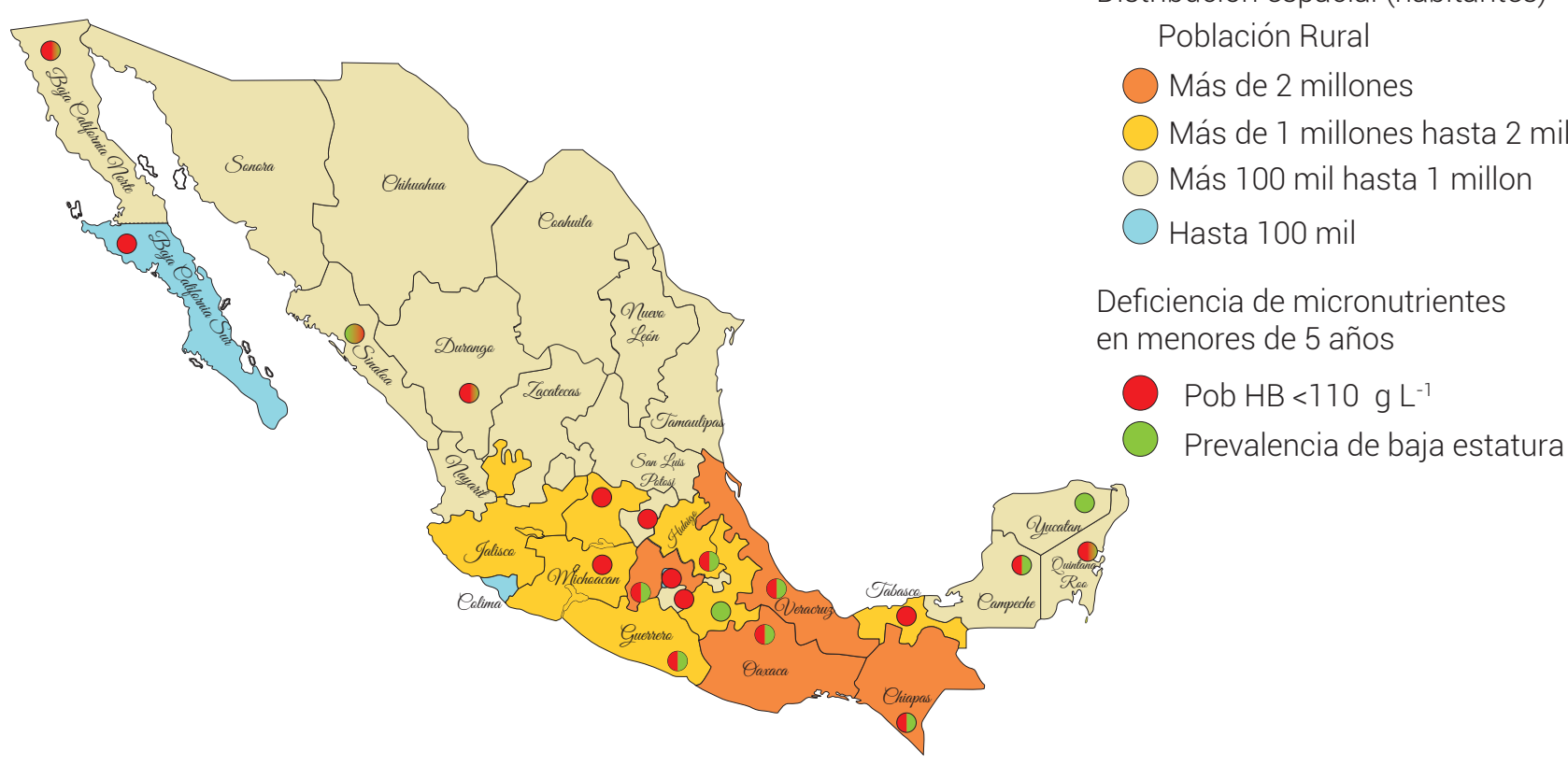

Figura 1. Distribución espacial de la población rural y deficiencia de micronutrientes. 
y Chiapas, con 400.3, 158.23, 120.40, 87.17, 63.98 y 60.34 miles de t; el rendimiento promedio nacional fue de $0.73 \mathrm{t}$ ha $^{-1}$ (SIAP, 2018). La producción nacional satisface cerca del $90 \%$ de las necesidades nacionales, por lo que en ese año se importaron 150 mil t de Estados Unidos, Canadá y Argentina (BNCE, 2019).

La comercialización de esta leguminosa está limitada de manera importante por las preferencias del consumidor; por ejemplo, en la región centro y sureste se consume principalmente frijol negro (FIRA, 2016). La producción de frijol negro se concentra sobre todo en Zacatecas, seguido de Chiapas, Oaxaca y Veracruz en el sureste del país; sin embargo, López-Salinas (2015), en un análisis de producción-consumo en la región sureste, determinó que desde hace más de una década la producción de frijol negro sólo cubre el $42.5 \%$ de las necesidades de su consumo y es abastecida por estados con superávit como Zacatecas, lo que refleja la movilidad del frijol
(Figura 2a). Según Yúnez y Barceinas (2000), ésto es debido en parte a las políticas de abastecimiento de años anteriores enfocadas a abastecer el consumo en zonas deficitarias que enmarcaron un patrón de consumo por tipo de frijol, además de las condiciones económicas. Con base en un modelo de distribución, García-Salazar et al. (2012) determinaron que una tercera parte de frijol pinto se consume en los estados productores y el restante en estados del noroeste y noreste (principalmente en Nuevo León y Tamaulipas), mientras que el consumo de frijol claro se presenta principalmente en centro occidente y centro, y en menor medida en el noroeste. Datos del SIAP (2018) indican que a nivel nacional indican el $87 \%$ de la oferta de frijol se destina a consumo humano, el restante a semilla, mermas y exportaciones.

Con respecto al consumo per capita anual de frijol, en los años 1980s se consumían alrededor de 20 kg por año, mientras que en la última década, se ha mantenido entre 9 (a)

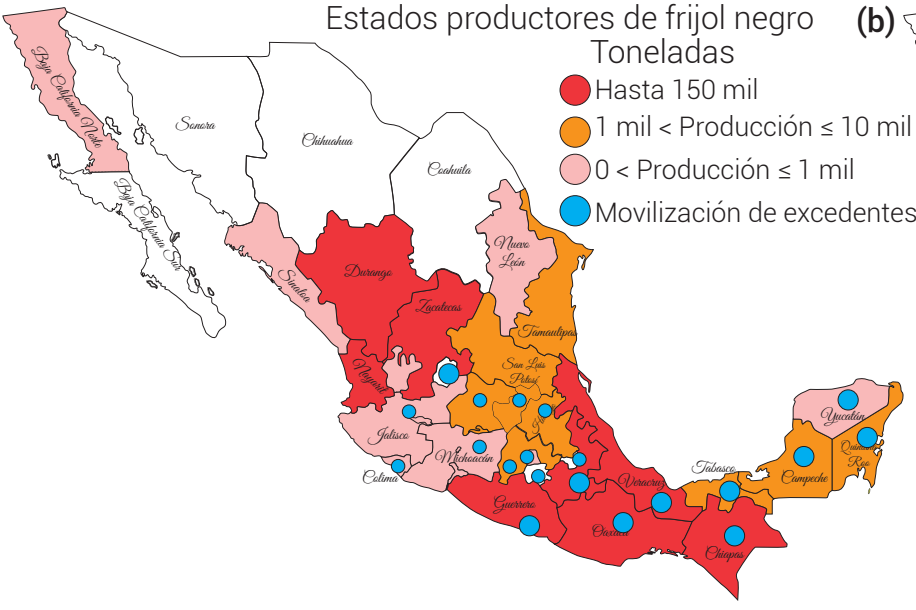

(b)

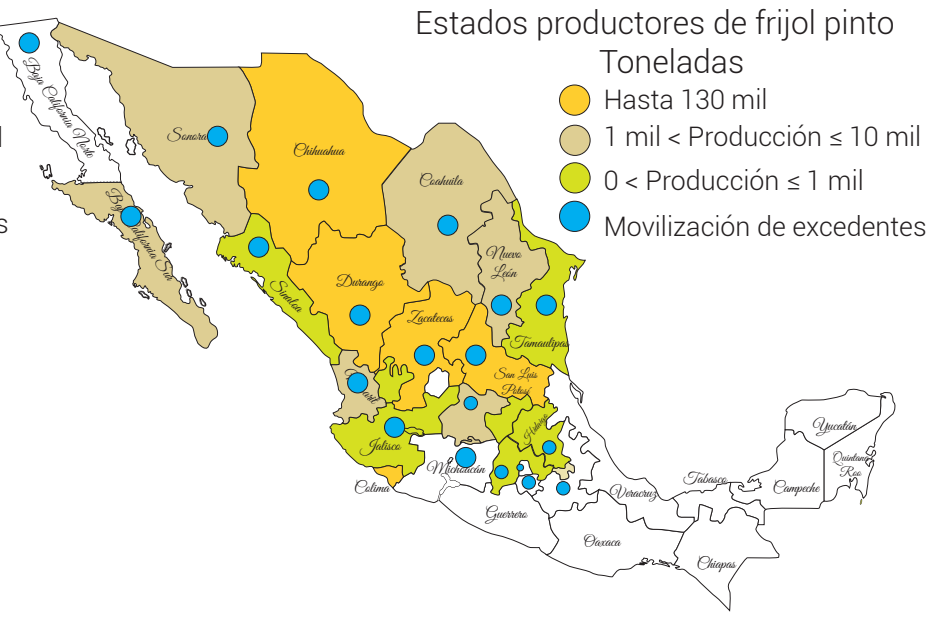

(c)

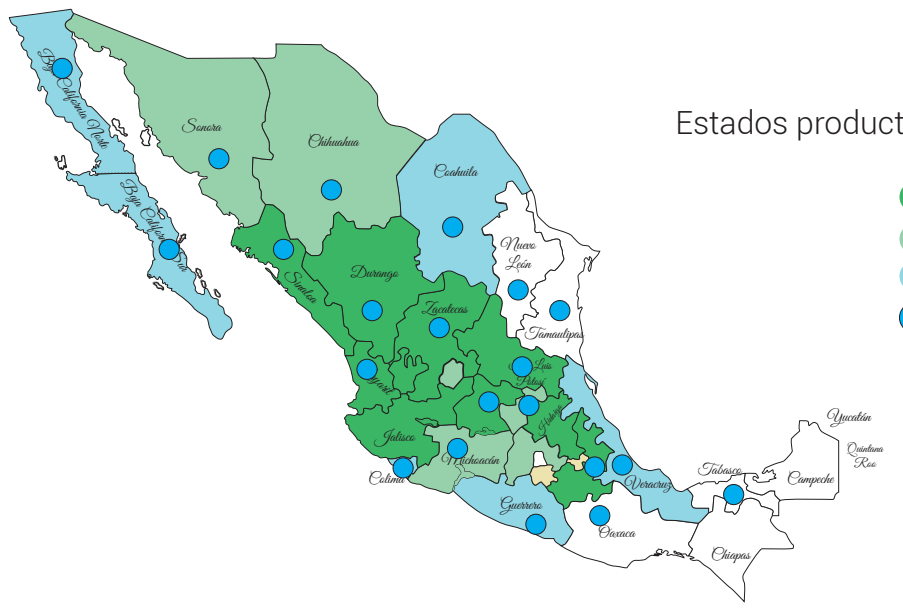

Figura 2. Distribución espacial de la producción y movilización de excedentes de frijol (a) negro, (b) pinto y (c) claro, según datos de García-Salazar et al. (2012). 
y $10 \mathrm{~kg}$ por año (FIRA, 2016). La tasa más alta de consumo se presenta en la población rural (que representa el $23 \%$ de la población nacional), la cual se concentra en estados como Oaxaca, Chiapas, Hidalgo, Guerrero, Zacatecas, Veracruz, San Luis Potosí, Michoacán y Durango (INEGI, 2010). El consumo de frijol en este sector es de $11.50 \mathrm{~kg}$ por año, mientras que en el urbano es de $5.73 \mathrm{~kg}$ por año; sin embargo, las recomendaciones de ingesta de frijol indican que el consumo en el sector rural debería de ser de 23.2 y en el urbano $18.2 \mathrm{~kg}$ por año para cumplir con los requerimientos de ingesta nutrimental (CONEVAL, 2012).

Una estrategia complementaria tendiente a incrementar los niveles de ingesta de hierro y zinc es la producción y consumo de cultivos básicos biofortificados; es decir, cultivos desarrollados a través de mejoramiento convencional, los cuales son competitivos agronómicamente y con mayores contenidos de micronutrientes. Desde el año 2004, el programa HarvestPlus trabaja en el desarrollo de nuevos cultivares básicos con mayor contenido de micronutrientes para combatir su deficiencia o hambre oculta en diferentes países de África, Asia y América Latina (Andersson et al., 2017). A la fecha, en Centroamérica, se han liberado variedades de frijol con más de $9 \mathrm{mg}$ de hierro100 $\mathrm{g}^{-1}$; es decir, $80 \%$ más de este micronutriente que las variedades tradicionales (Tello, 2017; Com. Pers.) ${ }^{1}$.

La implementación de este tipo de cultivos parte de priorizar áreas de intervención para maximizar el impacto nutricional según la producción, consumo y deficiencia de micronutrientes; así, el objetivo de esta investigación fue priorizar los estados del país para la intervención con frijol biofortifcado con hierro y zinc, mediante el Índice de Priorización para Biofortificación y las Condiciones de Biofortificación, con la finalidad de hacer recomendaciones de mejoramiento genético en cuanto a tipo de frijol y áreas de adaptación.

\section{MATERIALES Y MÉTODOS}

\section{Ámbito de estudio}

Se consideró como área total de estudio a la república mexicana y a cada uno de los 32 estados como subáreas. Se analizó el cultivo de frijol negro, claro y pinto. La estimación del Índice de Priorización de Biofortificación y las Condiciones de Intervención para Biofortificación (CIB) se realizaron a partir de los índices de producción, consumo y deficiencia de micronutrientes establecidos

\footnotetext{
'Tello F. G. (2017) ICTA Chortí, nueva variedad de frijol con más hierro y zinc para Guatemala. Coordinadora Disciplina de Divulgación, Instituto de Ciencia y Tecnología Agrícola. Guatemala. http:// lac.harvestplus.org/icta-chorti-frijol-biofortificado-guatemala/ (Marzo 2018)
}

por HarvestPlus desde 2013, los cuales parten de tres premisas: 1) producir y consumir al menos una parte de la producción, 2) un consumo per capita significativo de la producción interna del cultivo y 3) prevalencia considerable de la deficiencia de micronutrientes en la población (AsareMarfo et al., 2013; Herrington et al., 2019).

\section{Definición de los índices}

\section{Índice de producción (IP)}

Mide la magnitud de la producción del cultivo en cuestión en cada sub-área; las variables contempladas se refieren a la superficie cosechada de frijol, la superficie per capita cosechada del mismo, así como sus exportaciones; es decir, los volúmenes enviados a otras sub-áreas o fuera del área de estudio.

\section{Índice de consumo (IC)}

Se refiere al consumo del cultivo en cuestión que es producido en las sub-áreas a partir de las variables consumo per capita por año y la proporción de las importaciones.

Tanto el IP como el IC están diseñados para evitar sesgos derivados del tamaño en términos absolutos, como superficie sembrada o cosechada, tamaño de la población, volúmenes exportados o importados, etc.

\section{Índice de deficiencia de micronutrientes (IDM)}

Está conformado por el subíndice de deficiencia de hierro (SDH) y el subíndice de deficiencia de zinc (SDZ). El $\mathrm{SDH}$ mide el nivel de deficiencia a partir de las variables proporción de niños en edad preescolar con hemoglobina $(\mathrm{Hb})<110 \mathrm{~g} \mathrm{~L}^{-1}$ y la proporción de años de vida perdidos por deficiencia de hierro-anemia por cada 100,000 habitantes para México. El SDZ se determina a partir del porcentaje de Población en Riesgo de Ingesta Insuficiente de Zinc (\% PRII), con base en estudios antropométricos reportados por Brown et al. (2004), la proporción de grupos vulnerables de toma inadecuada de zinc (Ramírez-Jaspeado et al., 2018) y la prevalencia de talla baja (altura/edad) por estado, de niños entre los 0 y 60 meses de edad.

\section{Escalamiento de variables}

Mediante un enfoque heurístico, la adición matemática y la incorporación de variables con diferentes unidades de medición se basaron en el método de conversión utilizado en el Índice de Desarrollo Humano (HDI, por sus siglas en inglés) que permite escalar las variables (UNDP, 1990) y el Índice de Hambre Mundial (GHI por sus siglas 
en inglés GHI). Las unidades oscilan entre 0 y 1 , y son independientes de unidades de medida. El valor mínimo se refiere a la observación $n$ con menor valor del grupo de observaciones (de las 32 sub-áreas); para el caso de las variables porcentuales el intervalo es entre 0 y 100 (Ecuación 1).

Valor re-escalado $=\frac{\text { Valor actual }- \text { Valor mínimo }}{\text { Valor máximo }- \text { Valor mínimo }}$

Los valores mínimos y máximos de producción y consumo de frijol, además de la deficiencia de hierro y zinc en la población que se consideran para el cálculo se presentan en la Cuadro 1.
Para la estimación de los índices, las variables fueron reescaladas, y se denotan con el superíndice $r$, con base en la Ecuación 1 y los valores mínimos y máximos del Cuadro 1. El IP permite evaluar la producción consumida de manera doméstica mediante la suma ponderada de la superficie cosechada de frijol per capita y la tierra asignada al cultivo en la sub-área sujeta al término (1 - porcentaje de exportación). Las sub-áreas con producción nula de frijol consideran el valor de la adición como cero, por lo que el índice de frijol será cero (Ecuación 2).

$$
\begin{aligned}
I P_{(a)(b)}= & \left((1 / 2) \times \text { Área per capita cosechada }_{(a)(b)}{ }^{r}\right. \\
& \left.+(1 / 2) \times \text { Proporción del área cosechada }{ }_{(a)(b)}{ }^{r}\right) \\
& \times\left(1-\text { Proporción de exportaciones }_{(a)(b)}\right)^{2}
\end{aligned}
$$

\begin{tabular}{|c|c|c|c|c|c|c|}
\hline & Variable & Tipo & Mínimo & Sub-área & Máximo & Sub-área \\
\hline \multirow[t]{12}{*}{ Producción } & \multirow{4}{*}{$\begin{array}{l}\text { Participación de la superficie } \\
\text { cosechada (\%) }\end{array}$} & Negro & 0 & & 17.6 & Zacatecas \\
\hline & & Pinto & 0 & & 29.4 & Durango \\
\hline & & Claro & 0 & & 23 & Zacatecas \\
\hline & & Total & 0 & & 49.7 & Zacatecas \\
\hline & \multirow{4}{*}{$\begin{array}{l}\text { Superficie per capita cosechada } \\
\left(\mathrm{m}^{2}\right)\end{array}$} & Negro & 0 & & 1,336 & Zacatecas \\
\hline & & Pinto & 0 & & 1,214 & Durango \\
\hline & & Claro & 0 & & 1,752 & Zacatecas \\
\hline & & Total & 0 & & 3,779 & Zacatecas \\
\hline & \multirow[t]{4}{*}{ Participación de exportaciones (\%) } & Negro & 0 & & 86 & Zacatecas \\
\hline & & Pinto & 0 & & 78 & Durango \\
\hline & & Claro & 0 & & 83 & Zacatecas \\
\hline & & Total & 0 & & 83 & Zacatecas \\
\hline \multirow[t]{7}{*}{ Consumo } & \multirow{4}{*}{$\begin{array}{l}\text { Consumo per capita para } \\
\text { consumo humano (kg/año) }\end{array}$} & Negro & 0.38 & Chihuahua & 5.54 & Oaxaca \\
\hline & & Pinto & 0.18 & Campeche & 10.21 & N.L. \\
\hline & & Claro & 1.47 & Durango & 7.29 & Michoacán \\
\hline & & Total & 6.23 & Q. Roo & 14.34 & N.L. \\
\hline & \multirow[t]{3}{*}{ Proporción de importaciones (\%) } & Negro & & & & \\
\hline & & Pinto & 0 & & 100 & \\
\hline & & Total & & & & \\
\hline \multirow{2}{*}{$\begin{array}{l}\text { Subíndice de } \\
\text { deficiencia de } \\
\text { hierro }\end{array}$} & HbLess 110 (\%) & & 15 & Coahuila & 35 & Campeche \\
\hline & DALYS IDA & & 56 & Quéretaro & 311 & Tabasco \\
\hline \multirow{2}{*}{$\begin{array}{l}\text { Subíndice de } \\
\text { deficiencia de } \\
\text { zinc }\end{array}$} & Toma inadecuada de zinc (\%) & & 18.5 & Q. Roo & 20 & Oaxaca \\
\hline & $\begin{array}{l}\text { Prevalencia de talla baja } \\
\text { (altura/edad) (\%) }\end{array}$ & & 5 & Sonora & 31 & Chiapas \\
\hline
\end{tabular}

Cuadro 1. Valores máximo y mínimo de producción y consumo de frijol y deficiencias de hierro y zinc. 
donde (a): 32 estados de la república, (b): tipo de frijol (general, negros, pintos, claros).

El IC comprende sólo la proporción del consumo local referente a la producción de cada sub-área a partir del consumo per capita, sujeta al termino (1 - proporción de importaciones) (Ecuación 3).

$$
\begin{aligned}
I C_{(a)(b)}= & \text { Consumo humano per capita }{ }_{(a)(b)^{r}} \\
& \left(1 \text {-Proporción de importaciones }{ }_{(a)(b)}{ }^{r}\right)
\end{aligned}
$$

El IDM se calcula a partir de las Ecuaciones 4 y 5 mediante una media ponderada, donde el SDH presenta una participación de 70 \% y el SDZ $30 \%$.

$$
\begin{aligned}
& \mathrm{SDH}_{(\mathrm{a})}=(1 / 2) H b<110_{(\mathrm{a})}^{r}+(1 / 2) \mathrm{DALY} \text { per HDA }{ }_{(\text {a) }}^{r} \text { (Ec.4) } \\
& \begin{aligned}
\operatorname{SDZ}_{(a)} & =(1 / 2) \text { Toma inadecuada de } \operatorname{zinc}_{(a)}{ }^{r} \\
& \left.+(1 / 2) \text { Deficiencia de talla baja }{ }_{(a)}\right)^{\prime}
\end{aligned} \\
& \operatorname{IDM}_{(\mathrm{a})}=\left[(.70) \times \mathrm{SDH}_{(\mathrm{a})}\right]+\left[(.30) \times \mathrm{SDZ}_{(\mathrm{a})}\right]
\end{aligned}
$$

\section{Índice de priorización para biofortificación}

Permite identificar y clasificar los estados según el impacto potencial que puedan tener las intervenciones con frijol biofortificado en la reducción de las deficiencias de hierro y zinc, a partir de los tres subíndices calculados. Dada la alta correlación entre el IP e IC, se considera una media geométrica para ambos para asegurar una participación homogénea con el índice de micronutrientes. La Ecuación 7 presenta la priorización entre 0 y 1; para facilidad de exposición, la ecuación 7 se multiplica por 100 para expresar el IPB de 0 a 100. Para re-escalarlo se utiliza la Ecuación 1 (intervalo de 0 a 100).

$$
I P B_{(a)(b)}=\sqrt{\sqrt{I P_{(a)(b)} \times I C_{(a)(b)}} \times I D M_{(a)}}
$$

\section{Ajustes por superficie y población objetivo}

El IPB ajustado considera un ajuste por tamaño de superficie y otro por población objetivo. El ajuste de una sub-área en relación con el área de estudio se nombra como $A$ y varía en un intervalo de 0 a 1, lo que permite que el IPB de cualquier sub-área pueda ser ajustado. Los valores obtenidos de la Ecuación 8 pueden ir de 0 a cualquier número positivo. Con fines de comparación se debe aplicar el método para re-escalar (Ecuación 1) y multiplicar por 100 para obtener un valor entre 0 y 100.

$$
\left.I P B_{(a)(b)}\right)_{\text {ajustado }}=I P B_{(a)(b)}{ }^{r} \times A \times 100
$$

El ajuste por superficie se deriva de la importancia de generar prioridades de mejoramiento genético, así implique la producción de volúmenes importantes de semilla, y que la producción de frijol sea beneficiada por economías de escala que permitan reducir costos de producción y distribución; el ajuste está en función de la superficie cosechada de frijol de una sub-área con respecto a la totalidad de las sub-áreas consideradas. El segundo se refiere a la población objetivo de cada subárea respecto de la suma de la población objetivo total, donde la población objetivo es la población rural de niños de entre 0 y 5 años y mujeres entre 15 y 49 años.

\section{Condiciones de intervención para la biofortificación}

Se establecieron tres condiciones en función de su prioridad, donde los índices de producción, consumo y deficiencia de micronutrientes se delimitaron en importancia baja o alta a partir de la mediana de cada variable como punto de referencia (Ramírez-Jaspeado et al., 2018). Las condiciones son las siguientes:

$$
\begin{aligned}
& \text { Nivel } 7 \text { (Impacto e intervencion): } \\
& I C_{(a)(b)}>\operatorname{MeIC}_{(b)} ; I P_{(a)(b)}>\text { MeIP } P_{(b)} ; I D M_{(a)}>\text { MeIDM ... } \\
& \text { Nivel } 2 \text { (Impacto): } \\
& I C_{(a)(b)} \geq \operatorname{MelC}_{(b)} ; I P_{(a)(b)} \leq \operatorname{MeIP}(b) ; I D M_{(a)} \geq \operatorname{MeIDM~...~} \\
& \text { Nivel } 3 \text { (Intervención): } \\
& I C_{(a)(b)}<M e I C_{(b)} ; I P_{(a)(b)}>M e l P_{(b)^{\prime}} ; I D M_{(a)}<\operatorname{MeIDM} \ldots
\end{aligned}
$$

El Cuadro 2 muestra un resumen de las variables usadas para calcular los tres subíndices con sus respectivas fuentes de información. El periodo considerado para el análisis es de 2013 a 2015. Las exportaciones de grano por estado se determinaron en función de los volúmenes disponibles de producción excedentaria bajo el supuesto de exportar únicamente cuando la demanda ha sido satisfecha, y de la distribución espacial de los volúmenes movilizados entre los estados de frijol negro, claro y pinto obtenido de García-Salazar et al. (2012), mientras que las importaciones para estados deficitarios se determinaron a partir de la demanda de frijol menos su disponibilidad. Dichas variables son utilizadas para el cálculo de proporción de exportaciones e importaciones.

\section{RESULTADOS}

La priorización sin ajuste más alta se observó en las regiones agroclimáticas centro-occidente: Guanajuato y SLP; Chiapas, Oaxaca y Guerrero ubicados en la región sureste se priorizan con un índice de entre 60 y $72 \%$; y la región centro: Puebla e Hidalgo, con un índice de 70 \%. 
Cuadro 2. Variables utilizadas para generar los índices y subíndices.

\begin{tabular}{|c|c|c|c|}
\hline $\begin{array}{l}\text { Índices y } \\
\text { subíndices }\end{array}$ & Variable & Términos relacionados por sub-área & Principales fuentes \\
\hline \multirow[t]{3}{*}{ Producción } & $\begin{array}{l}\text { Participación de la } \\
\text { superficie cosechada (\%). }\end{array}$ & $\begin{array}{l}\text { Superficie cosechada de frijol (ha)/superficie } \\
\text { total agrícola del área de estudio (ha). }\end{array}$ & $\operatorname{SIAP}(2018)$ \\
\hline & $\begin{array}{l}\text { Superficie cosechada per } \\
\text { cápita }\left(\mathrm{m}^{2}\right)\end{array}$ & $\begin{array}{l}\text { Superficie cosechada de frijol (ha) /Población } \\
\text { del área de estudio (hab). }\end{array}$ & $\begin{array}{l}\text { SIAP (2018), INEGI (2010), } \\
\text { CONAPO (2015) }\end{array}$ \\
\hline & $\begin{array}{l}\text { Proporción exportaciones } \\
(\%)\end{array}$ & $\begin{array}{l}\text { Si la producción es más grande de } \\
\text { 0: la proporción de las exportaciones } \\
\text { =Exportaciones/(Producción +Importaciones); } \\
\text { por el contrario, la proporción de las } \\
\text { importaciones es } 0\end{array}$ & $\begin{array}{l}\text { BNCE (2019), } \\
\text { SIAP (2018) } \\
\text { BNCE (2019), } \\
\text { (SIAP, 2018) }\end{array}$ \\
\hline \multirow[t]{2}{*}{ Consumo } & $\begin{array}{l}\text { Consumo humano per } \\
\text { capita (kg/año) }\end{array}$ & Consumo humano per capita (kg/año) & $\begin{array}{l}\text { INEGI (2010), CONAPO } \\
\text { (2015), SIAP (2018), FIRA } \\
\text { (2016) }\end{array}$ \\
\hline & $\begin{array}{l}\text { Proporción de } \\
\text { importaciones (\%) }\end{array}$ & $\begin{array}{l}\text { Si la producción es más grande de 0: } \\
\text { la proporción de las importaciones = } \\
\text { Importaciones/ (Producción + Importaciones- } \\
\text { exportaciones); de lo contrario la proporción de } \\
\text { las importaciones es } 10 \% \text {. }\end{array}$ & $\begin{array}{l}\text { BNCE (2019) } \\
\text { SIAP (2018) }\end{array}$ \\
\hline \multirow{2}{*}{$\begin{array}{l}\text { Subíndice de } \\
\text { deficiencia de } \\
\text { hierro }\end{array}$} & Hb Less $110(\%)$ & $\begin{array}{l}\text { Proporción: niños en edad preescolar Hb }<110 \\
\mathrm{~g} \mathrm{~L}^{-1}\end{array}$ & Gutiérrez et al. (2012) \\
\hline & DALYS IDA & DALYs lost per 100,000 inhabitants to IDA & GBD (2016) \\
\hline \multirow{2}{*}{$\begin{array}{l}\text { Subíndice de } \\
\text { deficiencia de } \\
\text { zinc }\end{array}$} & $\begin{array}{l}\text { Toma inadecuada de zinc } \\
(\%)\end{array}$ & $\begin{array}{l}\text { Porcentaje de la Población en Riesgo de } \\
\text { Inadecuada ingesta de Zinc (\% PRTII) }\end{array}$ & Ramírez-Jaspeado (2018) \\
\hline & $\begin{array}{l}\text { Prevalencia de talla baja } \\
\text { (altura/edad) }(\%)\end{array}$ & $\begin{array}{l}\text { Prevalencia de talla baja (-2 DS por debajo de la } \\
\text { normal en niños de entre } 0 \text { y } 60 \text { meses de edad. }\end{array}$ & Gutiérrez et al. (2012) \\
\hline $\begin{array}{l}\text { Ajuste por } \\
\text { población }\end{array}$ & $\begin{array}{l}\text { Proporción de la población } \\
\text { rural objetivo (\%) }\end{array}$ & $\begin{array}{l}\text { Población rural objetivo (PO) (mujeres en edad } \\
\text { reproductiva y niños de } 0 \text { a } 59 \text { meses) (hab)/ PO } \\
\text { del área de estudio (hab). }\end{array}$ & $\begin{array}{l}\text { INEGI (2010) } \\
\text { CONAPO (2015) }\end{array}$ \\
\hline $\begin{array}{l}\text { Ajuste por } \\
\text { superficie }\end{array}$ & $\begin{array}{l}\text { Proporción de la superficie } \\
\text { dedicada a la producción de } \\
\text { frijol (\%). }\end{array}$ & $\begin{array}{l}\text { Superficie cosechada de frijol en la sub-área } \\
\text { (ha)/superficie total cosechada de frijol en el } \\
\text { área de estudios (ha). }\end{array}$ & $\operatorname{SIAP}(2018)$ \\
\hline
\end{tabular}

Por tipo de frijol, el IPB para frijol negro es más alto en la región sureste: Chiapas, Oaxaca, Guerrero, Veracruz, Campeche, Tabasco y Quintana Roo; en la región centro se priorizan los estados de Puebla e Hidalgo, mientras que Guanajuato se priorizó en la región centro-occidente. Respecto al IPB de frijol pinto, cinco de los 10 estados priorizados están en la región centro-occidente: Zacatecas, SLP, Nayarit, Guanajuato y Querétaro; sin embargo, Baja California Sur, ubicado en el Noroeste es el principal estado priorizado, y en tercer lugar Chihuahua en el noreste. En frijol claro, el IPB más alto se presenta en las regiones centrooccidente: Guanajuato, Querétaro, SLP, Aguascalientes y Nayarit; centro: Hidalgo, Tlaxcala y Puebla; y Noroeste: Sinaloa y Sonora (Cuadro 3).

\section{IPB ajustado por la población objetivo}

Al comparar IBP no ajustado (Cuadro 3) con el ajustado por Población Objetivo (Cuadro 4), se observa que la mayoría de los estados continuaron en las principales posiciones, excepto Durango, BCS y Nayarit, mientras que Veracruz pasó a la cuarta posición, al igual que el Estado de México y Michoacán en los lugares nueve y diez. Una variación importante se observa en Chiapas (al pasar de la posición ocho a la dos) y SLP (al pasar de la dos a la ocho), debido a que Chiapas presenta una población objetivo 2.8 veces más grande que SLP. Para frijol negro, el IPB ajustado se mantuvo en nueve de los 10 estados con mayor priorización respecto al no ajustado, Quintana Roo salió de las 10 primeras posiciones, mientras que el Estado 
de México pasó a la posición ocho; uno de los cambios más destacados es el de Campeche que pasó del lugar seis al 10, ya que concentra menos del $1 \%$ de la población objetivo total. En frijol pinto, los estados de BCS, Coahuila y Tamaulipas perdieron importancia y salieron de los 10 primeros lugares dentro del IPB ajustado. Guanajuato se colocó como el de mayor priorización; Hidalgo, Sinaloa y Jalisco se ubicaron en los lugares lugares 7, 9 y 10, respectivamente. En frijol claro ocho de los 10 estados se conservaron en las primeras posiciones, SLP presentó el mayor IBP ajustado; Tlaxcala y Aguascalientes disminuyeron en priorización respecto al IBP no ajustado.
El estado de México y Michoacán recobraron importancia al ocupar las posiciones cinco y diez, respectivamente (Cuadro 4).

\section{IPB ajustado por superficie}

Al comparar las principales posiciones de los estados en el IPB no ajustado (Cuadro 3) con el IPB ajustado por área (Cuadro 5) se observa que, a nivel general, Durango pasó de la posición cinco a la uno; Guanajuato y SLP se conservaron en las primeras posiciones; Chihuahua al igual que Sinaloa, escalaron más de diez posiciones al

Cuadro 3. Índice de Priorización para Biofortificación (IPB) por tipo de frijol (\%).

\begin{tabular}{|c|c|c|c|c|c|c|c|c|}
\hline \multirow{2}{*}{ \# } & \multicolumn{2}{|c|}{ General } & \multicolumn{2}{|c|}{ Frijol negro } & \multicolumn{2}{|c|}{ Frijol pinto } & \multicolumn{2}{|c|}{ Frijol claro } \\
\hline & Estado & IPB & Estado & IPB & Estado & IPB & Estado & IPB \\
\hline 1 & Guanajuato & 100.0 & Chiapas & 100.0 & BCS & 100.0 & Guanajuato & 100.0 \\
\hline 2 & SLP & 81.2 & Oaxaca & 86.8 & SLP & 89.9 & Hidalgo & 66.4 \\
\hline 3 & Puebla & 72.8 & Guerrero & 76.1 & Chihuahua & 67.0 & Querétaro & 66.2 \\
\hline 4 & Oaxaca & 72.3 & Puebla & 62.9 & Nayarit & 59.4 & Tlaxcala & 61.7 \\
\hline 5 & Durango & 71.9 & Veracruz & 61.2 & Sonora & 44.3 & Aguascalientes & 60.3 \\
\hline 6 & BCS & 71.0 & Campeche & 57.7 & Zacatecas & 43.8 & SLP & 56.0 \\
\hline 7 & Hidalgo & 69.7 & Tabasco & 54.1 & Coahuila & 28.8 & Puebla & 52.3 \\
\hline 8 & Chiapas & 62.8 & Guanajuato & 44.8 & Guanajuato & 28.2 & Sinaloa & 48.8 \\
\hline 9 & Nayarit & 62.3 & Hidalgo & 44.4 & Querétaro & 16.3 & Sonora & 48.3 \\
\hline 10 & Guerrero & 60.1 & Q. Roo & 41.1 & Tamaulipas & 14.7 & Nayarit & 43.4 \\
\hline
\end{tabular}

Cuadro 4. Índice de Priorización para Biofortificación (IPB) con ajuste por población, según el tipo de frijol (\%).

\begin{tabular}{|c|c|c|c|c|c|c|c|c|}
\hline \multirow{2}{*}{ \# } & \multicolumn{2}{|c|}{ General $^{+}$} & \multicolumn{2}{|c|}{ Frijol negro $^{+}$} & \multicolumn{2}{|c|}{ Frijol pinto $^{+}$} & \multicolumn{2}{|c|}{ Frijol claro ${ }^{+}$} \\
\hline & Estado & IPB & Estado & IPB & Estado & IPB & Estado & IPB \\
\hline 1 & Guanajuato & 100.0 & Chiapas & 100.0 & Guanajuato & 100.0 & SLP & 100.0 \\
\hline 2 & Chiapas & 91.7 & Oaxaca & 72.9 & SLP & 82.2 & Guanajuato & 64.7 \\
\hline 3 & Oaxaca & 81.7 & Veracruz & 70.2 & Zacatecas & 22.0 & Sinaloa & 42.7 \\
\hline 4 & Veracruz & 78.3 & Guerrero & 39.9 & Nayarit & 21.8 & Puebla & 36.1 \\
\hline 5 & Puebla & 69.0 & Puebla & 33.0 & Sonora & 17.2 & México & 27.2 \\
\hline 6 & Hidalgo & 51.6 & Tabasco & 22.2 & Chihuahua & 16.3 & Sonora & 25.8 \\
\hline 7 & Guerrero & 48.2 & Hidalgo & 14.5 & Hidalgo & 14.4 & Hidalgo & 25.8 \\
\hline 8 & SLP & 42.0 & México & 8.3 & Querétaro & 13.1 & Nayarit & 19.0 \\
\hline 9 & México & 31.0 & Guanajuato & 8.1 & Sinaloa & 9.0 & Querétaro & 16.8 \\
\hline 10 & Michoacán & 26.7 & Campeche & 5.2 & Jalisco & 7.8 & Michoacán & 16.8 \\
\hline
\end{tabular}

${ }^{\dagger}$ Ajuste (A) por población objetivo 
Cuadro 5. Índice de Priorización para Biofortificación (IPB) con ajuste por superficie, según el tipo de frijol (\%).

\begin{tabular}{|c|c|c|c|c|c|c|c|c|}
\hline \multirow{2}{*}{$\#$} & \multicolumn{2}{|c|}{ General $^{+}$} & \multicolumn{2}{|c|}{ Frijol negro $^{+}$} & \multicolumn{2}{|c|}{ Frijol pinto $^{+}$} & \multicolumn{2}{|c|}{ Frijol claro $^{+}$} \\
\hline & Estado & IPB & Estado & IPB & Estado & IPB & Estado & IPB \\
\hline 1 & Durango & 100.0 & Chiapas & 100.0 & Chihuahua & 100.0 & Guanajuato & 100.0 \\
\hline 2 & Guanajuato & 44.0 & Oaxaca & 39.9 & Zacatecas & 50.5 & Sinaloa & 71.1 \\
\hline 3 & SLP & 41.9 & Veracruz & 24.3 & SLP & 33.9 & SLP & 57.2 \\
\hline 4 & Chiapas & 38.8 & Puebla & 23.7 & Nayarit & 5.7 & Hidalgo & 24.4 \\
\hline 5 & Chihuahua & 31.8 & Guerrero & 12.9 & BCS & 2.5 & Puebla & 20.5 \\
\hline 6 & Puebla & 22.5 & Guanajuato & 10.4 & Coahuila & 1.5 & Nayarit & 16.7 \\
\hline 7 & Sinaloa & 21.2 & Hidalgo & 6.6 & Sonora & 1.2 & Querétaro & 11.3 \\
\hline 8 & Oaxaca & 16.1 & Durango & 5.8 & Guanajuato & 1.2 & Aguascalientes & 9.1 \\
\hline 9 & Nayarit & 16.0 & Nayarit & 5.0 & Tamaulipas & 0.1 & Jalisco & 6.7 \\
\hline 10 & Hidalgo & 13.0 & Tabasco & 2.6 & Jalisco & 0.1 & Tlaxcala & 5.3 \\
\hline
\end{tabular}

${ }^{\dagger}$ Ajuste (A) por superficie

colocarse en la número cinco y siete, respectivamente, mientras que BCS y Guerrero perdieron importancia al no colocarse en las primeras diez posiciones.

Por tipo de frijol, las posiciones más importantes en el IPB para frijol negro las ocuparon Chiapas, Oaxaca, Veracruz, Puebla, Guerrero, Guanajuato. En frijol pinto, se observó que Chihuahua y Zacatecas se colocaron en las dos primeras posiciones al ser dos de los tres estados que dedican mayor superficie al cultivo de esta leguminosa. BCS, SLP y Nayarit continuaron en las primeras posiciones; sin embargo, el valor de los índices no ajustados estuvo muy por encima de los ajustados. Querétaro perdió importancia (10 al 11) y Jalisco pasó al lugar 10. Con relación al índice ajustado de frijol claro, Guanajuato conservó la posición al dedicar parte importante de su superficie al cultivo de frijol. Sinaloa y SLP también se beneficiaron con el ajuste al subir a las primeras posiciones, Sonora perdió su posición en los primeros 10 lugares, mientras que Jalisco alcanzó el lugar nueve.

\section{Las Condiciones de Intervención para Biofortificación (CIB)}

Las CIB se determinaron para los tres tipos de frijol en los tres niveles de prioridad mencionados anteriormente. Para frijol negro biofortificado las áreas de impacto e intervención serían en la región sureste (seis de los siete estados considerados) y en la región centro-occidente (Guanajuato). Las áreas de impacto fueron BCS y Sonora en la región noroeste; Morelos y Tlaxcala en la región centro. Los estados considerados de prioridad baja se localizan en la región centro-occidente: Querétaro, con un bajo IC por un elevado porcentaje de importaciones y SLP, con un bajo consumo per capita de frijol, además de ser uno de los estados con menores problemas de HB, por debajo de $110 \mathrm{~g} \mathrm{~L}^{-1}$.

En el caso del frijol pinto, las áreas consideradas de impacto e intervención para la biofortificación son Sonora y BCS en el noroeste del país, así como Guanajuato en el centro-occidente, los cuales no se caracterizan por una elevada producción y consumo; sin embargo, la mayor parte de su producción permanece en sus respectivos estados; además, se estima que una tercera parte de su población rural menor de 5 años presenta niveles de HB por debajo de los $110 \mathrm{~g} \mathrm{~L}^{-1}$. Las áreas consideradas de impacto son Baja California en el noroeste, además de Morelos y Tlaxcala en la región centro. Las áreas de menor prioridad se centran en Jalisco y Querétaro en centro occidente; Hidalgo en la región centro y Nuevo León en el noreste. Cabe destacar que Nuevo León concentra $18 \%$ del consumo; sin embargo, su índice está por debajo de la mediana, derivado de una alta proporción de importaciones. Los estados de impacto e intervención para del frijol claro fueron Guanajuato en centro-occidente; Sinaloa y Sonora en el noroeste. Las áreas de impacto son Tamaulipas en el noreste y Tabasco en el sureste (Cuadro 6).

Se estratificó IPB (ajustado y sin ajustar) en tres grupos de importancia: elevada, media y baja en función de los estados 
Cuadro 6. Agrupación de los estados según las Condiciones de Intervención para Biofortificación.

\begin{tabular}{|c|c|c|c|}
\hline Tipo & Áreas de impacto e intervención ${ }^{+}$ & Áreas de impacto ${ }^{++}$ & Áreas de intervención ${ }^{++t}$ \\
\hline General & Oaxaca, Tlaxcala, Guanajuato, BCS & Tamaulipas, Sinaloa, Sonora & QRoo \\
\hline Negro & $\begin{array}{l}\text { Chiapas, Oaxaca, Guerrero, } \\
\text { Veracruz, Campeche, Tabasco, } \\
\text { Guanajuato }\end{array}$ & Morelos, Tlaxcala, BCS, Sonora & SLP y Querétaro \\
\hline Pinto & BCS, Guanajuato, Sonora & Tlaxcala, Morelos, BC & Hidalgo, Jalisco, Querétaro, NL \\
\hline Claro & $\begin{array}{l}\text { Tlaxcala, Guanajuato, Sinaloa, } \\
\text { Sonora }\end{array}$ & Tabasco, Tamaulipas & Durango, Chihuahua \\
\hline
\end{tabular}

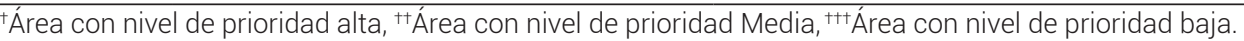

Cuadro 7. Estratificación del IPB en tres grupos de importancia de prioridad de acuerdo con su magnitud.

\begin{tabular}{llccc}
\hline \multirow{2}{*}{ Tipo de frijol } & Tipo de ajuste & \multicolumn{3}{c}{ Prioridad } \\
\cline { 2 - 4 } & Sin ajuste & Alta & Media & $<18.04$ \\
Negro & $>44.75$ & $18.04-44.75$ & $<1.10$ \\
& Por población objetivo & $>8.30$ & $1.10-8.30$ & $<0.88$ \\
& Por área & $>5.70$ & $0.88-5.70$ & $<16.30$ \\
Pinto & $>43.81$ & $16.30-43.81$ & $<9.00$ \\
& Sin ajuste & $>16.27$ & $9.00-16.27$ & $<0.10$ \\
& Por población objetivo & $>1.48$ & $0.10-1.48$ & $<33.73$ \\
Claro & Por área & $>48.80$ & $33.73-48.80$ & $<7.10$ \\
& Sin ajuste & $>19.03$ & $7.10-19.03$ & $<2.70$ \\
\hline
\end{tabular}

con un IPB mayor que 0, como se muestra en el Cuadro 7. Las coincidencias entre el IPB de importancia elevada (sin ajustar) y la condición de impacto e intervención, ambas para frijol negro, coinciden en que estados del sureste como Chiapas, Oaxaca, Guerrero, Veracruz, Campeche y Tabasco pueden verse beneficiados por la priorización con frijol negro biofortificado. Campeche y Tabasco se caracterizan por una población rural estatal por arriba de la media nacional, con pequeñas unidades de producción (2.2 ha) (INEGI, 2007), por lo que buena parte se destina al autoconsumo; además, presentan tasas de deficiencia por arriba de la media de uno u ambos micronutrientes. No existen coincidencias en el nivel medio, mientras que en el nivel de prioridad baja se considera a Querétaro tanto en el índice sin ajustar (Figura 1) como en el ajustado por población, y Jalisco se considera respecto al índice ajustado por área. Para frijol pinto, el IPB de prioridad elevada y la CIB de impacto e intervención concuerdan en que BCS y Sonora deberían ser prioritarios, mientras que Guanajuato y Sonora coinciden con la CIB en el índice ajustado por población; sin embargo, este último presenta baja importancia en el IPB. Para frijol claro, tanto el IPB sin ajustar con prioridad elevada, así como la condición de impacto e intervención concuerda en que Guanajuato y
Tlaxcala deberían ser prioritarios (Figura 3).

En Centroamérica, las variedades de frijol biofortificadas liberadas hasta la fecha son de frijol negro (Guatemala) y pinto (Nicaragua y Honduras). Las variedades liberadas como biofortificadas deben presentar niveles de hierro superiores los $7.5 \mathrm{mg} 100 \mathrm{~g}^{-1}$ de grano crudo, así como ser superiores a sus testigos en algunas cualidades agronómicas. En el caso de la variedad ICTA-Chortí de Guatemala, ésta presenta buen rendimiento y resistencia a la sequía; en el caso la variedad SMR88 de Nicaragua, tiene tolerancia a condiciones húmedas y enfermedades (Valdivia, 2017); mientras que Honduras Nutritivo se caracteriza por su excelente potencial de rendimiento y adaptación a ambientes diversos de producción de frijol, su resistencia a los Virus del Mosaico Dorado Amarillo del Frijol (VMDAF) y al Virus Mosaico Común del Frijol (VMCF) (SAGH, 2016), y son variedades aptas principalmente para zonas bajas (abajo de $1600 \mathrm{msnm}$ ).

Por las características de consumo, se recomienda evaluar para México primeramente variedades de color negro ya liberados, así como germoplasma existente en el Centro Internacional de Agricultura Tropical (CIAT) que 
(a)

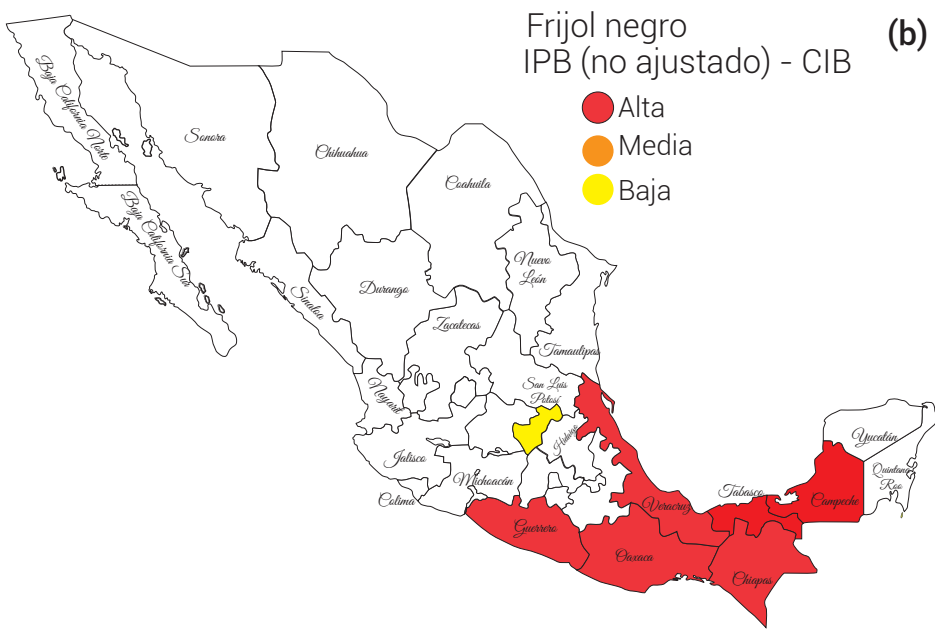

(b)

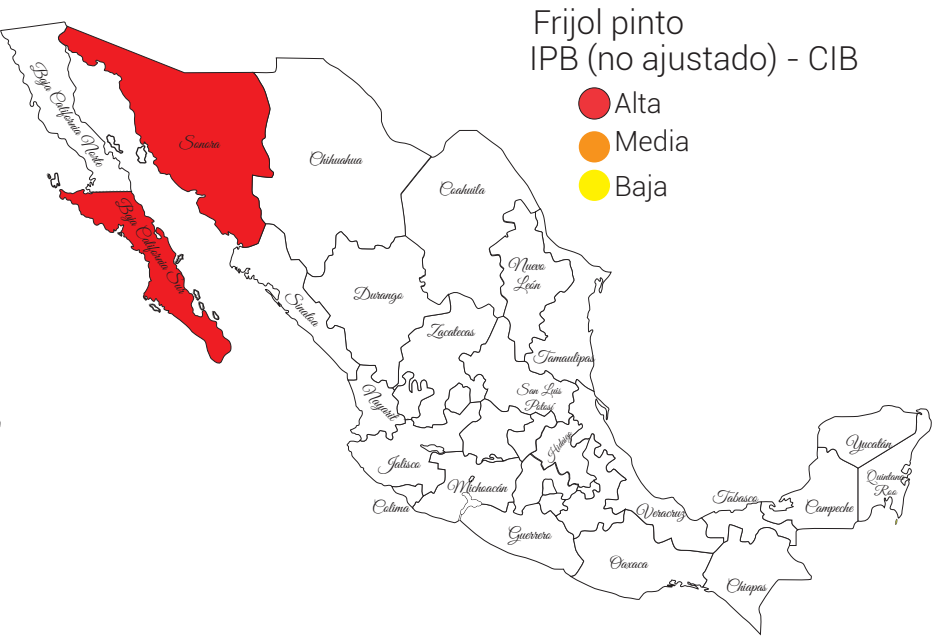

(c)

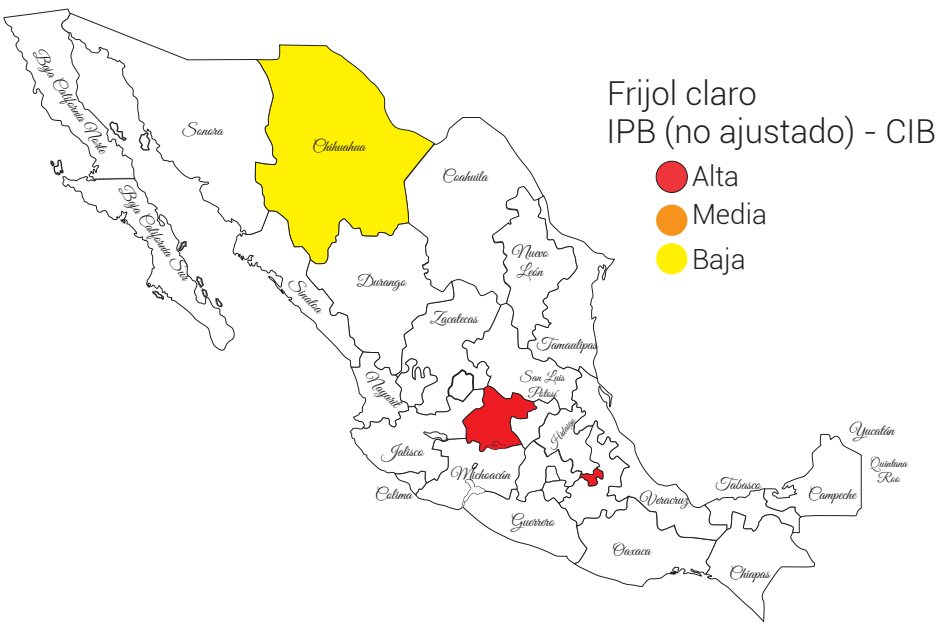

Figura 3. Índice de priorización para biofortificación (IPB) y condiciones de intervención para biofortificación (CIB) para frijol negro (a), pinto (b) y claro (c) en México.

puedan potencializar la evaluación agronómica en los estados prioritarios aquí identificados: Chiapas, Oaxaca, Guerrero, Veracruz, Campeche y Tabasco, poniendo especial énfasis en enfermedades como VMDAF, VMCF y hoja angular, que reducen el rendimiento de frijol de manera significativa. Para el caso de frijol pinto, donde se destaca la priorización de los estados de Sonora y BCS, se deberían iniciar trabajos referentes a biofortificación de variedades nativas o nacionales (las variedades pintas biofortificadas existentes no son consumidas en México) y centrarse en la tolerancia a la sequía y resistencia a enfermedades como antracnosis, roya y tizón común que atacan las plantaciones de frijol en la región semiárida del país. Adicionalmente, sería de importancia continuar los estudios con frijol biofortificado en México reportados por Márquez-Quiroz et al. (2018) que incluyen un mayor número de evaluaciones en contenidos de micronutrientes, rendimiento agronómico, tolerancia a enfermedades, adaptación y características de calidad de grano que demandan los consumidores.
Finalmente, es necesario reconocer que aunque IPB y CIB representan una aproximación válida para la intervención con cultivos biofortificados (Asare-Marfo et al., 2013), la falta de desagregación de algunos datos como el consumo (determinado nivel nacional), limitan la interpretación.

\section{CONCLUSIONES}

Los resultados del índice de priorización para biofortificación (IPB) sin ajuste sugieren que estados del sureste como Chiapas, Oaxaca, Guerrero, Veracruz, Campeche y Tabasco deberían ser priorizados para la intervención con frijol negro, tanto el escenario de ajuste de población objetivo como de superficie refuerzan estos resultados. Para frijol pinto, BCS y Sonora son los principales estados prioritario, mientras que en frijol claro Guanajuato es altamente prioritario tanto por el IPB con y sin ajustes, así como para las condiciones de intervención para biofortificación (CIB); Tlaxcala también es señalado 
como de prioridad tanto en el IPB como para las CIB.

\section{AGRADECIMIENTOS}

Soporte financiero parcial para este estudio fue proporcionado por HarvestPlus (www. HarvestPlus.org), una alianza global de instituciones de investigación en agricultura y nutrición que trabajan para incrementar la densidad de micronutrientes en cultivos básicos. Los puntos de vista aquí expresados no necesariamente reflejan los de HarvestPlus. El consorcio de investigación CRP-MAIZE también apoyo parcialmente este trabajo.

\section{BIBLIOGRAFÍA}

Andersson M. S., A. Saltzman, P. S. Virk and W. H. Pfeiffer (2017) Progress update: crop development of biofortified staple food crops under HarvestPlus. African Journal of Food, Agriculture, Nutrition and Development 17:11905-11935.

Asare-Marfo D., E. Birol, C. González, M. Moursi, S. Pérez, J. Schwarz and M. Zeller (2013) Prioritizing Countries for Biofortification Interventions Using Country-Level Data. International Food Policy Research Institute. Washington, D. C. 44 p.

BNCE, Barra Nacional de Comercio Exterior (2019) Estadísticas por fracción arancelaria. Barra Nacional de Comercio Exterior. Ciudad de México. http://www.barradecomercio.org/?p=2571 (Febrero 2019).

Brown K. H., J. A. Rivera, Z. Bhutta, R. S. Gibson, J. C. King, B. Lönnerdal, ... and C. Hotz (2004) International Zinc Nutrition Consultative Group (IZiNCG) Technical Document \#1. Assessment of the risk of zinc deficiency in populations and options for its control. Food and Nutrition Bulletin 25(Suppl. 2):S99-S203.

CONABIO, Comisión Nacional para el Conocimiento y Uso de la Biodiversidad (2012) Frijol. Comisión Nacional para el Conocimiento y Uso de la Biodiversidad. México, D. F. https://www.biodiversidad.gob. mx/usos/alimentacion/frijol.html (Enero 2018).

CONAPO, Consejo Nacional de Población (2015) Proyecciones de la Población de México y de las Entidades Federativas, 2016-2050. Consejo Nacional de Población. Ciudad de México. https:// datos.gob.mx/busca/dataset/proyecciones-de-la-poblacionde-mexico-y-de-las-entidades-federativas-2016-2050 (Febrero 2018)

CONEVAL, Consejo Nacional de Evaluación de la Política de Desarrollo Social (2012) Construcción de las Líneas de Bienestar. Documento Metodológico. Consejo Nacional de Evaluación de la Política de Desarrollo Social. México, D. F. 79 p.

De los Santos-Ramos M., T. Romero-Rosales y E. E. Bobadilla-Soto (2017) Dinámica de la producción de maíz y frijol en México de 1980 a 2014. Agronomía Mesoamericana 28:439-453, https://doi.org/10.15517/ma.v28i2.23608

Espinoza-García N., R. Martínez-Martínez, J. L. Chávez-Servia, A. M. Vera-Guzmán, J. C. Carrillo-Rodríguez, E. Heredia-García y V. A. Velasco-Velasco (2016) Contenido de minerales en semilla de poblaciones nativas de frijol común (Phaseolus vulgaris L.). Revista Fitotecnia Mexicana 39:215-223.

FAO, Organización de las Naciones Unidas para la Alimentación y la Agricultura (2017) El Estado de la Seguridad Alimentaria y la Nutrición en el Mundo 2017. Fomentando la Resiliencia en Aras de la Paz y la Seguridad Alimentaria. FAO, FIDA, OMS, PMA y UNICEF. Roma. $144 \mathrm{p}$.

FIRA, Fideicomisos Instituidos en Relación con la Agricultura (2016) Panorama Agroalimentario. Frijol 2016. Dirección de Investigación y Evaluación Económica y Sectorial. FIRA Banco de México. Ciudad de México. 36 p.

García-Salazar J. A., G. Rodríguez-Licea, M. Borja-Bravo y E. Guzmán-Soria (2012) Distribución espacial de la producción de frijol en función de las variedades demandadas por el consumidor. Paradigma Económico 4:85-99.
GBD, Global Burden of Disease (2016) Iron-deficiency anemia. DALYs (Disability-Ajusted Life Years) lost per 100,000. Institute for Health Metrics and Evaluation. University of Washington Seattle, Washington, USA http://ghdx.healthdata.org/gbdresults-tool (January 2018).

Gutiérrez J. P., J. Rivera-Dommarco, T. Shamah-Levy, S. VillalpandoHernández, A. Franco, L. Cuevas-Nasu, M. Romero-Martínez y M. Hernández-Ávila (2012) Encuesta Nacional de Salud y Nutrición 2012. Resultados Nacionales. Instituto Nacional de Salud Pública. Cuernavaca, Morelos, México. 196 p.

Herrington C., K. Lividini, M. D. Angel and E. Birol (2019) Prioritizing Countries for Biofortification Interventions: Biofortification Priority Index. 2nd edition. International Food Policy Research Institute. Washington, D. C. 64 p.

INEGI, Instituto Nacional de Estadística y Geografía (2007) Censo agrícola, ganadero y forestal 2007. Unidades de producción con cultivos en el ciclo primavera-verano y otoño-invierno. Instituto Nacional de Estadística y Geografía. Aguascalientes, México. https://www.inegi.org.mx/programas/cagf/2007/default html\#Tabulados (Marzo 2018).

INEGI, Instituto Nacional de Estadística y Geografía (2010) Censo de población y vivienda 2010. Instituto Nacional de Estadística y Geografía. Aguascalientes, México. https://www.inegi.org.mx/ programas/ccpv/2010/default.html\#Tabulados (Marzo 2018).

INSP, Instituto Nacional de Salud Pública (2012) Encuesta Nacional de Salud y Nutrición 2012. Resultados por entidad federativa. Instituto Nacional de Salud Pública Cuernavaca, Morelos. https://www.insp.mx/produccion-editorial/novedadeseditoriales/3057-ensanut2012-resultados-entidad-federativa. html (Enero 2018).

Latham M. C. (2002) Nutrición Humana en el Mundo en Desarrollo. Organización de las Naciones Unidas para la Alimentación y la Agricultura. Roma. $546 p$

Leyva L. N., E. P. Gutiérrez G., L. X. López M. y J. Basilio H. (2016) El frijol algo más que proteína. Ciencia y Desarrollo 42:50-55.

López-Salinas E., Ó. H. Tosquy-Valle, B. Villar-Sánchez, J. A. Acosta-Gallegos, J. R. Rodríguez-Rodríguez y P. Andrés-Meza (2015) Rendimiento y estabilidad de líneas mejoradas de frijol negro en Veracruz y Chiapas, México. Revista Fitotecnia Mexicana 38:173-181.

Márquez-Quiroz C., E. De-la-Cruz-Lázaro, R. Osorio-Osorio y E. SánchezChávez (2015) Biofortification of cowpea beans with iron: iron's influence on mineral content and yield. Journal of Soil Science and Plant Nutrition 15:839-847, https://doi.org/10.4067/ S0718-95162015005000058

Márquez-Quiroz C., E. de-la-Cruz-Lázaro, R. Osorio-Osorio, E. SánchezChávez, J. J. Huijara-Vasconcelos y J. Sida-Arreola (2018) Contenido de zinc y rendimiento de frijol caupí biofortificado Revista Mexicana de Ciencias Agrícolas 20:4175-4185, https://doi.org/10.29312/remexca.v0i20.988

Martínez M. M. R., A. Rojas, A. Santanen and F. L. Stoddard (2013) Content of zinc, iron and their absorption inhibitors in Nicaraguan common beans (Phaseolus vulgaris L.). Food Chemistry 136:87-93, https://doi.org/10.1016/j.foodchem.2012.07.105

Moraghan J. T. and K. Grafton (2001) Genetic diversity and mineral composition of common bean seed. Journal of the Science of Food and Agriculture 81:404-408, https://doi.org/10.1002/10970010(200103)81:4<404::AID-JSFA822>3.0.CO;2-H

Olivares G. M. y T. Walter K. (2003) Consecuencias de la deficiencia de hierro. Revista Chilena de Nutrición 30:226-233, https://doi.org/10.4067/S0717-75182003000300002

OMS, Organización Mundial de la Salud (2011) Concentraciones de hemoglobina para diagnosticar la anemia y evaluar su gravedad. Organización Mundial de la Salud. Ginebra, Suiza. 7 p. http://www.who.int/vmnis/indicators/haemoglobin_es.pdf (Abril 2018).

OMS, Organización Mundial de la Salud (2018) Malnutrición. Organización Mundial de la Salud. Ginebra, Suiza. https://www.who.int/es/ news-room/fact-sheets/detail/malnutrition (Abril 2018)

Prieto-Cornejo M. R., J. A. Matus-Gardea, F. Gavi-Reyes, J. M. Omaña-Silvestre, J. J. Brambila-Paz, J. Sánchez-Escudero y M. Á. Martínez-Damián (2019) Evolución de la superficie cultivada de frijol e impacto económico de la sequía sobre su rendimiento bajo condiciones de temporal en México. Revista Fitotecnia Mexicana 42:173- 
182, https://doi.org/10.35196/rfm.2019.2.173-182

Prolla I. R. D., R. G. Barbosa, A. P. L. Veeck, P. R. Augusti, L. P. da Silva, N. D. Ribeiro and T. Emanuelli (2010) Cultivar, harvest year, and storage conditions affecting nutritional quality of common beans (Phaseolus vulgaris L.) Ciência e Tecnología de Alimentos 30(Supl.1):96-102, https://doi.org/10.1590/S010120612010000500016

Ramírez-Jaspeado R., N. Palacios-Rojas, J. Funes, S. Pérez y M. L. Donnet (2018) Identificación de áreas potenciales en México para la intervención con maíz biofortificado con zinc. Revista Fitotecnia Mexicana 41:327-337.

SAGH, Secretaria de Agricultura y Ganadería de Honduras (2016) Primera variedad de frijol biofortificado es liberada en Honduras. Secretaria de Agricultura y Ganadería de Honduras. Tegucijalpa, Honduras. http://www.sag.gob.hn/sala-de-prensa/noticias/ano-2016/ abril-2016/primera-variedad-de-frijol-biofortificado-es-liberadaen-honduras-/ (Abril 2018)

SIAP, Servicio de Información Agroalimentaria y Pesquera (2018) Anuario estadístico de la producción agrícola. Servicio de Información Agroalimentaria y Pesquera. SAGARPA, Ciudad de México. http://nube.siap.gob.mx/cierreagricola/ (Mayo 2018)

UNDP, United Nations Development Programme (1990) Human Development Report 1990. Oxford University Press. New York USA. $141 \mathrm{p}$.

Valdivia, A. (2017) SMR88: La nueva variedad de fríjol liberada en Nicaragua. Centro Internacional de Agricultura Tropical. Cali, Colombia. https://blog.ciat.cgiar.org/es/smr88-la-nuevavariedad-de-frijol-liberada-en-nicaragua/ (Diciembre 2017).

WHO, World Health Organization (2013) Global database on child growth and malnutrition. World Health Organization of the United Nations. Geneve, Switzerland. http://www.who.int/ nutgrowthdb/database/countries/who_standards/mex_dat. pdf?ua $=1$ (Febrero 2018)

Yúnez N. A. y F. Barceinas (2000) Efectos de la desaparición de la Conasupo en el comercio y en los precios de los cultivos básicos. Estudios Económicos 15:189-227. 